\title{
Anaemia epidemiology in the era of "big data". Are we aware that the revolution is already going on?
}

\author{
Lucia Del Vecchio ${ }^{1}$
}

Received: 18 November 2019 / Accepted: 17 December 2019 / Published online: 8 January 2020

(c) Italian Society of Nephrology 2020

\section{Introduction}

More than 850 million people are affected by kidney disease worldwide [1]. According to projections, in 2040 chronic kidney disease (CKD), will be the 5th leading cause of years of life lost [2].

Anaemia is a frequent complication. Several observational studies have clearly shown association between anaemia and negative outcomes but, by definition, they cannot prove a causative relationship. Moreover, they suffer from the bias that anaemic patients are often sicker and at high risk for mortality or hospitalisation. Accordingly, randomised, clinical trials did not demonstrate a reduced risk of events when aiming at higher haemoglobin ( $\mathrm{Hb})$ levels with erythropoiesis stimulating agents (ESA).

\section{Is there still a role for observational studies?}

Safety concerns surrounding ESA therapy had urged new clinical guidelines suggesting lower $\mathrm{Hb}$ target to aim at with ESA $[3,4]$. This has also led to less therapeutic propensity to detect and treat anaemia. As a consequence, Hb levels have dropped significantly, with a significant proportion of CKD patients who are nowadays untreated or undertreated. In this evolving scenario, observational studies can give us new information on the clinical, social and economic impact of anaemia.

There are several approaches to data collection and analyses.

Community population studies randomly select and recruit individuals from a defined population in their community to receive extensive examinations and follow them for decades. This approach collects huge ton of data, but

Lucia Del Vecchio

luciadelvecchio@yahoo.com

1 Como, Italy possible biases exist on their applicability to broader populations and, by definition, they cannot collect information of those who refuse to participate. Example are the Atherosclerosis Risk in Communities Study or the National Health and Nutrition Examination Survey (NHANES).

Another possibility is to join together information from cohorts around the world. The Chronic Kidney Disease Prognosis Consortium (CKD-PC) performs collaborative meta-analyses to study CKD outcome. It collects over 70 cohorts from general, high-risk, or CKD populations [5].

Registry data have a long tradition in the nephrology. They opened the way to the awareness of CKD and allowed comparison on patient characteristics, renal replacement therapy (RRT) and outcome over time and among countries. However, they have often a limited set of information available, which is not necessarily uniform among countries. Data entry is often made on a voluntary basis, increasing the likelihood of missing data.

The Dialysis Outcomes and Practice Patterns Study (DOPPS) is a prospective cohort study; it collects observational, longitudinal data from a random sample of patients and facilities in twenty countries. Started on haemodialysis (HD), it has expanded to peritoneal dialysis and non-dialysis (ND)-CKD. Recently, anaemia prevalence has been compared in four different countries in ND-CKD [6].

Administrative data are more and more analysed with scientific purposes. In the United States, where the health care is provided by many distinct organisations and health care facilities are largely private, several for-profit and non-profit organisations made available their data base for observational research. This approach has given useful information about anaemia in CKD. However, they refer to selected parts of the population; selection biases can occur around social and economic strata of the population or peculiar characteristics for admission to the health care facility. For instances, the Veterans Health Administration covers only male patients. The United States Renal Data System (USRDS) largely collaborates with Medicare and Medicaid Services. 
Its nationwide coverage of the CKD population is incomplete, especially in ND-CKD. According to the 2018 Annual Report, $21 \%$ of HD patients had haemoglobin levels $<10 \mathrm{~g} /$ dl with $18.5 \%$ being treated with ESA therapy [7].

Toft et al. [8] presented the findings of a population-based study performed in Northern Denmark on anaemia and clinical outcomes in CKD. Data were obtained linking Danish population-based healthcare and administrative databases. The cumulative search population was of around two million persons, from which 16,972 CKD patients stage IV-V were identified with a total of 28,510 anaemia patient profiles. In this unselected population, being severely anaemic increased significantly the hazard ratio for acute hospitalisation and death from any cause. Among individual cardiovascular events, heart failure had the strongest association with anaemia.

\section{Do these findings add new knowledge?}

The paper by Toft et al. [8] is in line with previous findings coming from older cross-sectional studies [9]. Given its longitudinal design, it allows to detect also the incidence of anaemia over time and its burden on outcomes. Surprisingly, in this unselected, real-world context, half of the subjects had $\mathrm{Hb}$ levels $<10 \mathrm{~g} / \mathrm{dl}$.

The importance of the paper goes beyond its findings, since it represents a good example of a new type of clinical research. First, testing the prevalence and outcome of a given disease on unselected, large populations allows to better foresee related costs and clinical needs. Second, these data may assist clinicians to better characterise the severity of CKD and direct intensity of investigation and care. Third, the data might improve identification of individuals for entry into studies examining progression and outcome of CKD across a variety of clinical settings and laboratory abnormalities.

Even if Toft et al. [8] did not report data on ESA therapy, iron and blood transfusions, more in general administrative database contain information on drug prescription and on their actual consumption through selling reports. This gives unique opportunity to verify prescription strategies in everyday clinical practice and their true impact on patient outcomes. This is of particular importance in the field of nephrology. Despite the large prevalence of CKD worldwide and its burden on health status, patients with advanced CKD are often considered too complicate and fragile to be included in clinical trials. Given that many drugs are eliminated by the kidney, CKD patients display an increased risk of unanticipated adverse events. Moreover, treatment at reduced doses not necessarily has the same effects than the full dose.

Despite their completeness and continuous updating, data from unselected populations need to be interpreted cautiously.
Their final output largely depends on how the variables of interest are selected. New ways are needed to create data collection fields that make them more searchable also for scientific purposes. Particular cautions should be put in place to guarantee data security, storage and privacy protection.

Finally, these data have an economical value and can be used for commercial purposes. This has not necessarily a negative connotation. The analyses of big data require advanced software, hardware and statistical knowledge, which can be costly and not necessarily available in an administrative context. From one side, the health system can receive funding from allowing analyses of part of its dataset and use it to finance other activities. As a counterpart, pharmaceutical companies can be partners of health systems, obtain information that can be used to understand how many patients have the indication to a given treatment or track its selling. More importantly, the same data can become an asset available for the scientific community for independent research.

To conclude, in the last decade the world is going through the so-called fourth industrial revolution; it is characterized by a fusion of technologies mixing together the physical, digital, and biological spheres. Big data, artificial intelligence and deep learning are entering in many fields and changing them forever. In medicine they will open unthinkable opportunities, leading to new hypotheses and new ways to testing them. From an evidenced-base medicine, which gives recommendations for patient populations, we are moving towards personalised medicine, with algorithms able to generate the best treatment options for the single patient. This is not merely a dream but something within our reach. Recently a deep learning approach was developed for continuous risk prediction for future development of acute kidney injury starting from data from electronic health records [10]. The model could predict $55.8 \%$ of all inpatient episodes of acute kidney injury.

And then? Will doctors and nurses have no role in this new world? Definitely not. They will have at hand sophisticated tools for patient diagnosis and treatment and possibly have additional time to focus on patient needs and give a more complete meaning to the word "take care".

\section{Compliance with ethical standards}

Conflicts of interest Dr Lucia Del Vecchio had been member of Advisory Boards for DOC, Roche, Astellas, and invited speaker at meetings supported by DOC, Roche, Astellas, Vifor Pharma, Mudipharma. She is national leader for the ASCEND-ND study supported by GSK.

Ethical approval This article does not contain any study with human participants. 


\section{References}

1. Jager KJ, Kovesdy C, Langham R, Rosenberg M, Jha V, Zoccali C (2019) A single number for advocacy and communication-worldwide more than 850 million individuals have kidney diseases. Nephrol Dial Transplant 34(11):1803-1805.

2. Foreman KJ, Marquez N, Dolgert A et al (2018) Forecasting life expectancy, years of life lost, and all-cause and cause-specific mortality for 250 causes of death: reference and alternative scenarios for 2016-40 for 195 countries and territories. Lancet 392(10159):2052-2090

3. KDIGO (2012) Clinical practice guideline for anemia in chronic kidney disease. Kidney Int Suppl 2(4):279-335.

4. Locatelli F, Bárány P, Covic A et al (2013) Kidney disease: improving global outcomes guidelines on anaemia management in chronic kidney disease: a European Renal Best Practice position statement. Nephrol Dial Transplant 28:1346-1359

5. Inker LA, Grams ME, Levey AS, CKD Prognosis Consortium (2019) Relationship of estimated gfr and albuminuria to concurrent laboratory abnormalities: an individual participant data metaanalysis in a global consortium. Am J Kidney Dis 73(2):206-217.

6. Wong MMY, Tu C, Li Y et al (2019) Anemia and iron deficiency among chronic kidney disease stages $3-5$ ND patients in the chronic kidney disease outcomes and practice patterns study: often unmeasured, variably treated. Clin Kid J. https://doi.org/10.1093/ ckj/sfz091

7. Available at: https://www.usrds.org/adr.aspx last accessed on the 16th on November, 2019

8. Toft G, Heide-Jørgensen U, van Haalen H et al (2019) Anemia and clinical outcomes in patients with non-dialysis dependent or dialysis dependent severe chronic kidney disease: a Danish population-based study. J Nephrol. https://doi.org/10.1007/s4062 0-019-00652-9

9. Astor BC, Coresh J, Heiss G, Pettitt D, Sarnak MJ (2006) Kidney function and anemia as risk factors for coronary heart disease and mortality: the Atherosclerosis Risk in Communities (ARIC) Study. Am Heart J 151(2):492-500

10. Tomašev N, Glorot X, Rae JW et al (2019) (2019) A clinically applicable approach to continuous prediction of future acute kidney injury. Nature 572(7767):116-119

Publisher's Note Springer Nature remains neutral with regard to jurisdictional claims in published maps and institutional affiliations. 